\title{
IRECIST Unconfirmed Progressive Disease
}

National Cancer Institute

\section{Source}

National Cancer Institute. iRECIST Unconfirmed Progressive Disease. NCI Thesaurus.

Code C142360.

Apparent radiographic progression which requires confirmation, per criteria, with additional imaging. 